AGRICULTURE AND BIOLOGY JOURNAL OF NORTH AMERICA

ISSN Print: 2151-7517, ISSN Online: 2151-7525, doi:10.5251/abjna.2010.1.6.1303.1308

(C) 2010, ScienceHuß, http://www.scihub.org/ABJNA

\title{
Physiological studies on the production of exopolysaccharide by Fungi
}

\author{
Nehad, E.A. and A.R.El-Shamy \\ Department of Chemistry of Natural and Microbial Products, \\ National Research Center, Dokki, Egypt
}

\begin{abstract}
Six strains of microorganisms were screened for Exopolysaccharide production. Alternaria alternata was recognized to produce high levels of the EPS. The effects of environmental parameters on polysaccharide production by Alternaria alternate were investigated. Results showed the optimum temperature and initial $\mathrm{pH}$ for exopolysaccharide (EPS) production in shake flask cultures of Alternaria alternate were found to be $30^{\circ} \mathrm{C}$ and $\mathrm{pH} 3.0$, respectively. Incubation period required for maximum production was 9days. glucose was found to be the best carbon source to production of EPS, various concentrations of glucose were applied for the screening of the suitability of EPS production. 4\% glucose was favorable to production of EPS. Yeast extract gave the highest amount of EPS by Alternaria alternate, its maximum concentration for EPS was determined.
\end{abstract}

Keywords: Alternaria alternate, EPS production, physiological conditions

\section{INTRODUCTION}

Fungi are currently of interest because they are a biologically rich source of various active substances. Polysaccharides have emerged as an important class of bioactive substances.

Many types of polysaccharides could be produced by submerged cultures of higher fungi including mushrooms. The polysaccharide have been studied and used for pharmaceutical purposes due to their diverse biological activities (Song etal.,1998; Kim etal.,2005; Cui etal.,2003). These include antitumor and immunomodulating activities (Tokunaka etal.,2000; Bohn and Bemiller, 1995). Their mechanism has also been elucidated (Tsukada etal.,2003; Stanley etal.,2005). Most of the polysaccharides mediating biological activities from mycelia were endopolysaccharides (PPS) or exopolysaccharides (EPS) (Cheung,1996).

Exopolysaccharides are high molecular weight polymers of monosaccharides $(>20)$ and are secreted by a microorganism into the surrounding environment. Microorganisms synthesize a wide spectrum of multifunctional polysaccharides including intracellular polysaccharides, structural polysaccharides and extracellular polysaccharides or exopolysaccharides (EPS).

Exopolysaccharides have found multifarious applications in various food and pharmaceutical industries. Many microbial EPS provide properties that are almost identical to the gums currently in use. With innovative approaches, efforts are underway to supersede the traditionally used plant and algal gums by their microbial counterparts. Moreover, considerable progress has been made in discovering and developing new microbial EPS that possess novel industrial significance (Suresh and Mody, 2009).

The main purpose of this research point is screening the available local fungal strains for their EPS producing ability and select the most potent isolate. Also, to determine the optimum of environmental conditions on the Production of Exopolysaccharide by Alternaria alternate in submerged cultures.

\section{MATERIALS AND METHODS}

Organism:Six isolates of fungi namely (Alternaria alternate, Cladosporium herbarum, Acremonium charticela, Fusarium solani, Penicillium paraphergal and Gliomastic gueg ) were obtained from the Culture Collection unit from Department of Chemistry of Natural and Microbial Products at the National Research Center (NRC). Cultures were maintained on potato dextrose agar slopes. Slants were inoculated and incubated at $30^{\circ} \mathrm{C}$ for 7 days, and stored at $4^{\circ} \mathrm{C}$.

Shake flask culture: Shake flask cultures were carried out in $250 \mathrm{ml}$ Erlenmeyer flasks containing $50 \mathrm{ml}$ of medium. The media consisting of the 
following components (in $\mathrm{g} / \mathrm{l}$ ): glucose, 40; yeast extract, 1.0; peptone $0.5 ; \mathrm{KH}_{2} \mathrm{PO}_{4} \quad 0.5$; $\mathrm{MgSO}_{4} .7 \mathrm{H}_{2} \mathrm{O} 0.5$. Media were sterilized at $121^{\circ} \mathrm{C}$ for $20 \mathrm{~min}$. The $\mathrm{pH}$ was adjusted to 6.5 . The flasks were incubated on a rotary shaker at $28{ }^{\circ} \mathrm{C}$ and $150 \mathrm{rpm}$ for 2 weeks.

Extraction and assay of EPS: The mycelia biomass was seperated from the liquid medium by centrifugation (4000 rpm , 15min) and the supernatant was filtered through a Whatman filter paper No.1. The supernatant was collected and mixed with 5 volumes of $95 \%$ ethanol ( $/ \mathrm{v})$ and left overnight at $4^{\circ} \mathrm{C}$ for polysaccharide precipitation. The precipitate was collected as the crude EPS fraction. The concentration of EPS was determined by Phenol-sulfuric acid method (Chaplin and Kenned ,1986). The absorbane of the sample was measured at $490 \mathrm{~nm}$ and calibrated to total sugar content using glucose as a standard.

Optimization of the culture conditions: To evaluate maximum production of crude EPS produced by fungi, The following criteria were studied.

a. Incubation period:

The culture media are incubated for different incubation periods $3,4,7,9,10$ and 14 days at $30^{\circ} \mathrm{C}$ on an incubator shaker 150rpm

b. Initial pH :

The selected fungal isolate cultivated in fermentation medium at different $\mathrm{pH}$ values $(3.0,4.0,5.0,6.0,7.0,8.0$ and 9.0$)$ at $30^{\circ} \mathrm{C}$.

c. Temperature:

The fungal culture was incubated at $26,28,30$, 32 , and $35^{\circ} \mathrm{C}$.

d. Carbon sources:

Different carbon sources were examined for the production of EPS. Those carbon source were glucose, molasses, starch, lactose, fructose, soucrose, xylose and cellulose.

e. Nitrogen sources

Different nitrogen sources were examined for the production of EPS.

Those nitrogen source were yeast extract, peptone, corn steep liquor, malt extract, beef extract tryptophane urea, casein, soybean and $\mathrm{NaNO}_{3}$ as a sole sources of nitrogen.

\section{RESULTS AND DISCUSSION}

Production of Exopolysaccharide (EPS) by different fungal strains:The purpose of this experiment was to study the best fungal strain which produce high level of EPS, we used six different strains from the culture collection unit of NRC namely Alternaria alternate, Cladosporium herbarum, Acremonium charticela, Fusarium solani, Penicillium paraphergal and Gliomastic gueg.

The results in Fig. (1) showed that Alternenia alternate was the best Strain for the production of EPS (6.58 mg/ml). Also Yang and Liau, (1998); Gao and Gu, (2007); Leung etal., (2009) produced EPS from Gonoderma lucidum, Aganicus brasiliensis, Cordyceps sinensis, respectively.

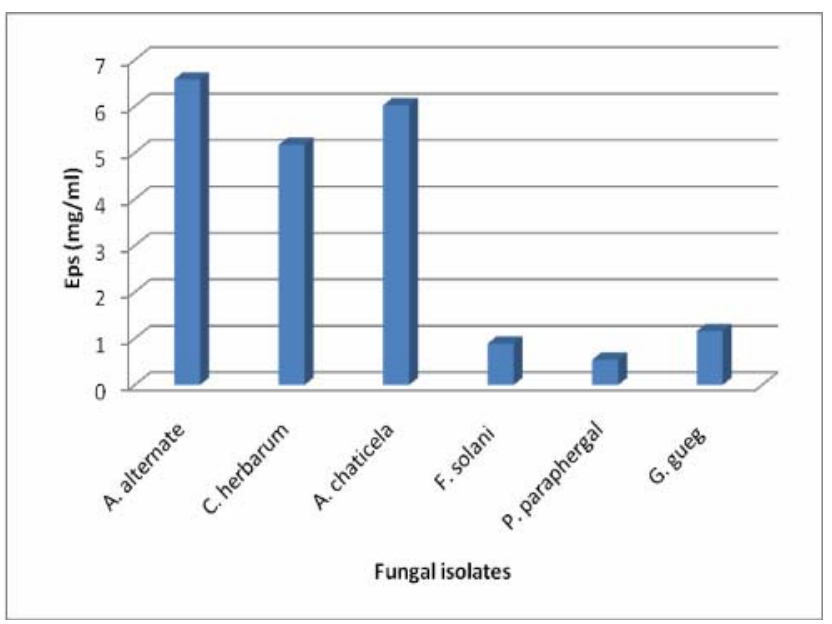

Fig 1: production of Exopolysaccharide (EPS) by different fungal strains

\section{Optimization of the fermentation conditions:}

Effect of incubation period on (EPS) production by Alternania alternata: Production of EPS during different incubation periods was studied by A.alternate using a culture medium consist of the following components (ing/l): glucose, 40; yeast extract, 1.0; peptone $0.5 ; \mathrm{KH}_{2} \mathrm{PO}_{4} \quad 0.5$; $\mathrm{MgSO}_{4} \cdot 7 \mathrm{H}_{2} \mathrm{O}$ 0.5. The results in Fig. (2) showed the expolysaccharide accumulation by Alterneria altermata in the basal medium of shake cultures. We observed a rapid increase in the exopolysaccharide concentration within the first 9 days. The exopolysaccharide concentrations were the highest at day 9 and give a corresponding maximum value $11.96 \mathrm{mg} / \mathrm{ml}$ after that this values decreased. Thus we performed all of the following fermentations for 9 days, optimizing the condition in the flask culture. Rong etal., (2010) found that 4 days incubations is 
the best for exopolysaccharide production with Hirsutella pat.

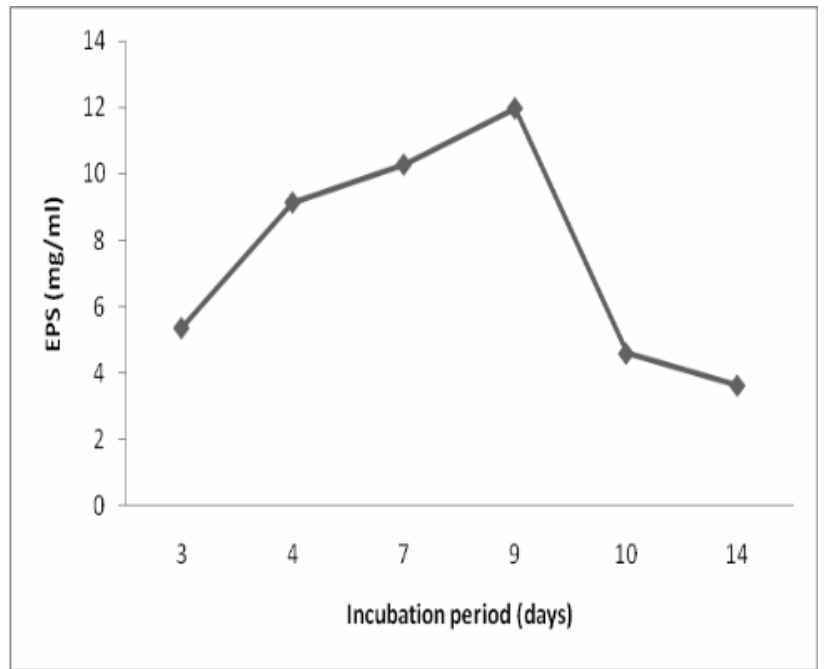

Fig 2: The effect of different incubation periods on Exopolysaccharide (EPS) production by Alternaria alternate

Effect of intial PH on (EPS) production by Alternania alternate: The $\mathrm{pH}$ of the culture medium is a vital factor that governs mycelial growth and exopolysaccharide production.

The results in Fig. (3) showed that the optimal pH for exopolysaccharide production was 3 and at higher values of $\mathrm{pH}$ the exopolysaccharide production declined sharply, other researchers have a reported, optimum $\mathrm{pH}$ of 5.5 for other exopolysaccharide synthesizing fungi such as Pleurotus pulmonarius (Nour et al, 2004).

Rong et al.,( 2010) found that the optimum $\mathrm{pH}$ for exopolysaccharide by Hirsultella pat is 5.5

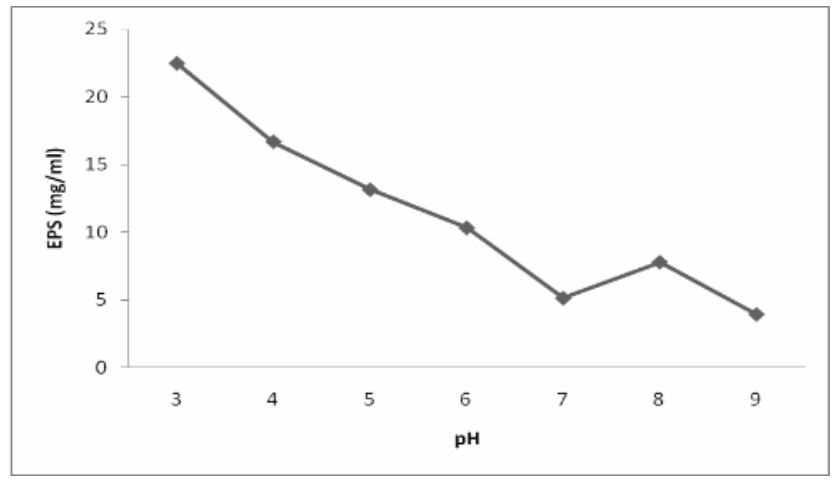

Fig 3: The effect of initial $\mathrm{PH}$ value of the medium on Exopolysaccharide (EPS) production by Alternaria alternate

Effect of different temperature on (EPS) production by Alternania alternate:The results in Fig. (4) shows that the optimal temperature for polysaccharide formation by Alternaria alternate was found to be $30^{\circ} \mathrm{C}$. The production rate of polysaccharide decreased rapidly above this value.

(Yang and Liau, 1998) proved that arang between $30^{\circ} \mathrm{C}$ and $33^{\circ} \mathrm{C}$ was found to be suitable for EPS production by Gonoderma lucidum.

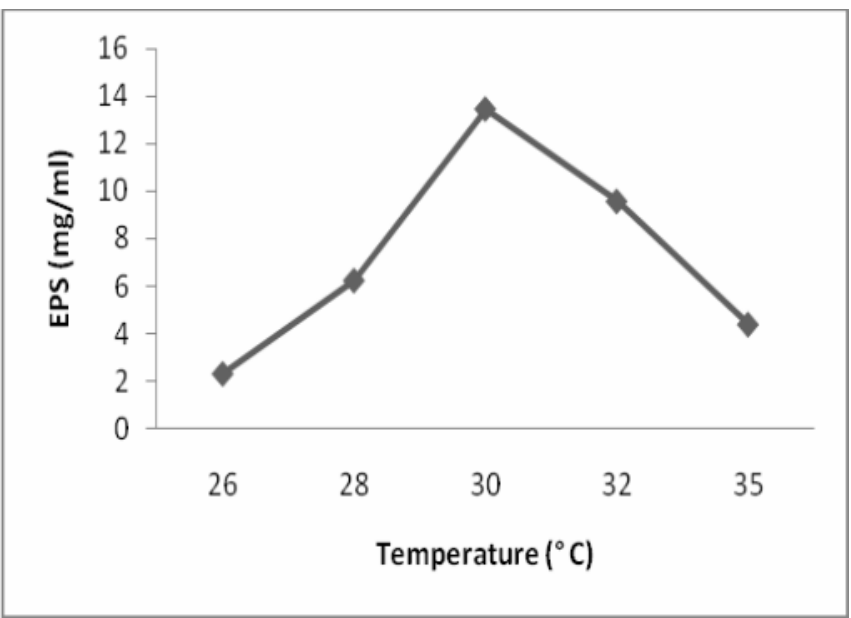

Fig 4: Effect of temperature on production of Exopolysaccharide (EPS) by Alternaria alternate

Effect of different carbon source of on EPS production_by Alternaria alternate: Carbohydrates are a major component of the cytoskeleton and an important nutritional requirement for the growth and development of higher fungi (Xiao et al., 2006).

The effects of carbon sources on the production of EPS were studied in the fermentation medium containing various carbon source. The results in Fig. (5) showed that glucose is the best carbon source for ESP production using Alternaria alternate giving $16.3 \mathrm{mg} / \mathrm{ml}$ followed by Xylose which gave $14.6 \mathrm{mg} / \mathrm{ml}$. it was reported that sucrose the most suitable carbon source for mycelium growth production in Cordyceps militaris (Park et al., 2001), while glucose was selected for Phellinus gilures (Hwang et al., 2003; Xu et al., 2003). Maltose was also known as an efficient carbon source for EPS production by mushrooms (Bae et al., 2000; Wu et al., 2003). Starch was chosen as carbon source for Pleurotus tuberregium 
production. Various concentration of glucose (1-5\%) were applied for the screening of the suitability of polysaccharide production. 4\% glucose was significantly desirable at achieving maximum production of EPS (Fig. 6).

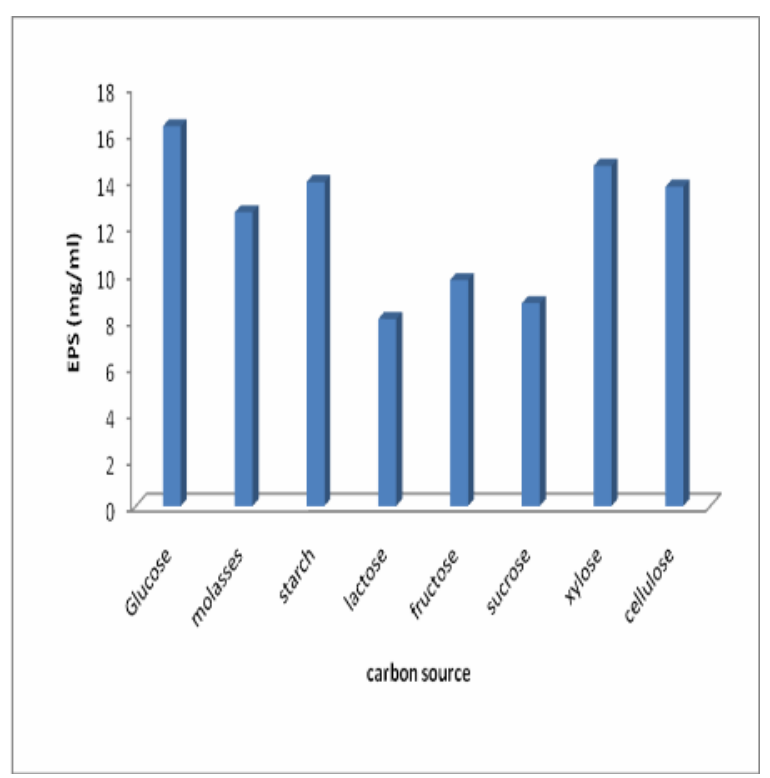

Fig 5: Effect of different carbon sources on EPS production by Alternaria alternate

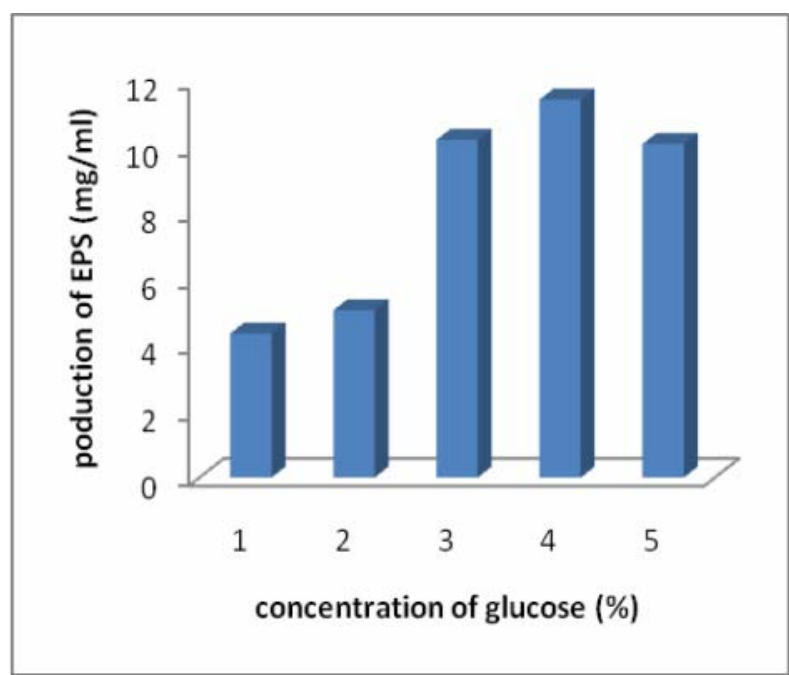

Fig 6: Effect of different concentration of glucose on EPS production by Alternaria alternat
Effect of different nitrogen sources on EPS production by Alternaria alternate: As illustrated in Fig. (7) the effects of nitrogen sources on the production of EPS were studied in the fermentation medium containing various nitrogen sources. Yeast extract followed by $\mathrm{NaNO}_{3}$ were the best sources of nitrogen for EPS production giving 25.3 and 22.13 $\mathrm{mg} / \mathrm{ml}$ respectively, the stimulatory effect of yeast extract is due to its protein, amino acid, and vitamin content (Bolton and Blain, 1982). The highest level of EPS were obtained when peptone was used as a sole nitrogen source by Hirsutella sp. (Rong et al., 2010).

Various concentrations of yeast extract, $0.5 \%, 1 \%$, $1.5 \%$ and $2 \%$ were applied to identify the suitable concentration for polysaccharides production. A yeast extract of $2 \%$ concentration stimulate the maximum EPS production $(4.5 \mathrm{mg} / \mathrm{ml})$. This results were in agreement with (Pokhrel and Ohga, 2007) who proved that $2 \%$ yeast extract was the best for maximum EPS production by lyophyllum decastes , pleurotus pulmonarius.

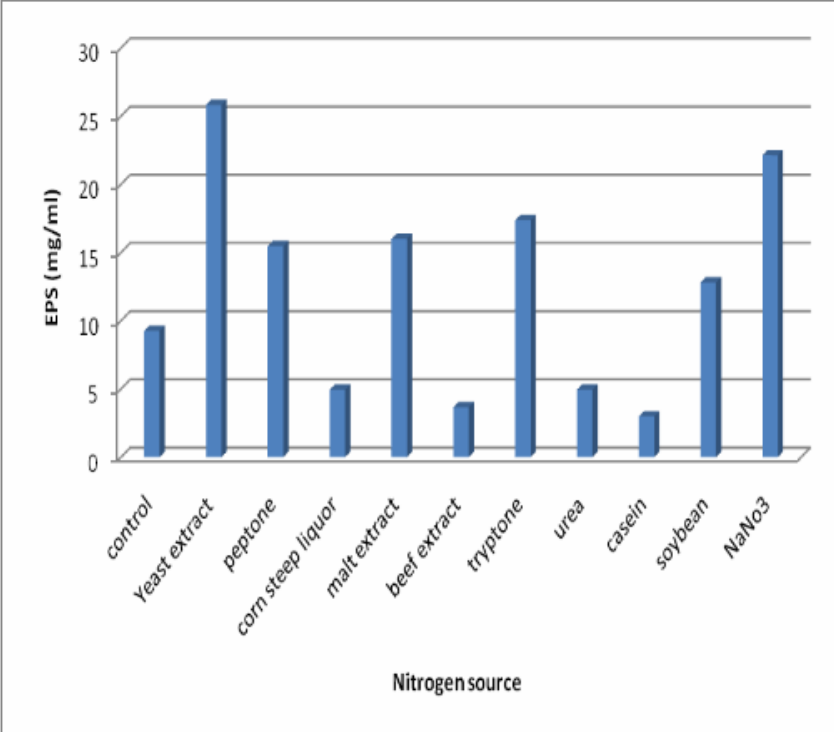

Fig. 7: Effect of different nitrogen sources on EPS production by Alternaria alternate 


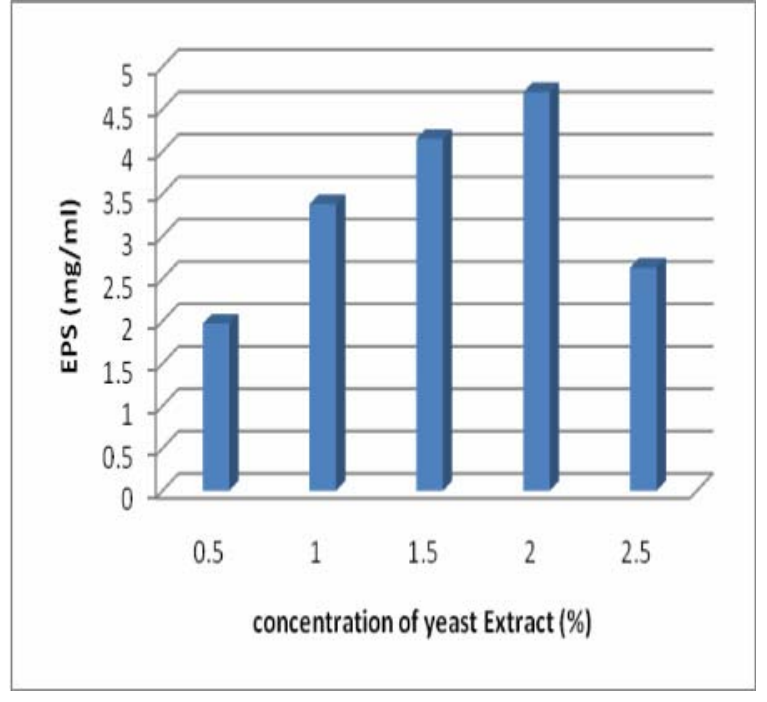

Fig. 8: Effect of different concentration of yeast Extract on EPS production by Alternaria alternate.

\section{CONCLUSIONS}

Alternaria alternate was selected from six types of fungi for maximum production of Exopolysaccharides. Our study concentrated on selecting a suitable duration time, nutrient source, their concentrations, initial $\mathrm{pH}$ and temperature.

\section{REFERENCES}

Suresh and Mody (2009). "Microbial Exopolysaccharides: Variety and Potential Applications". Microbial Production of Biopolymers and Polymer Precursors. Caister Academic Press. ISBN 978-1-904455-36-3.

Song, C.H.; Jeon, Y.J.; Ra KS, Kim HI. (1998). Anticomplementary activity of endopolymers produced from submerged mycelial culture of higher fungi with particular reference to Lentinus edodes. Biotechnol Letter, 20:741-4.

Kim, Y.O.; Han, S.B.; Lee, H.; Ahn, H.J.; Yoon, Y.D.; Jung, J.K.(2005). Immunostimulating effect of the endopolysaccharide produced by submerged culture of Lnonotus obliquus. Life Sci., 77:2438-56.

Cui. J.; Chisti, Y. (2003). Polysaccharides of Coriolus versicolor: physiological activity, use and production. Biotechnol Adv.; 21:109- 22.

Tokunaka, K.; Ohno, N.; Adachi ,Y.; Tanaka, S.; Tamura ,H.; Yadomae T.(2000). Immunopharmacological and immunotoxicological activities of a watersoluble (13)d-glucan CSBG from Candida spp. Int J. Immunopharm ;22:383-94.
Bohn ,J.A.; Bemiller, J.N.(1995). (1-3) d-Glucans as biological response modifiers: a review of structure functional activity relationships. Carbohydrate Polym.; 28:3-14.

Chaplin,M.F. and Kennedy, J.F. (1986). Carbohydrate analysis: Apartical Approach oxford university press, Oxford,UK, pp.1-2.

Tsukada C, Yokoyama H, Miyaji C, Ishimoto Y, Kawamura H, Abo T.(2003). Immunopotentiation of interaepithelial lymphocytes in the intestine by oral administrations of glucan. Cell Immunol.; 221:1-5.

Stanley. G.; Harvey, K.; Slivova.; Jiang.; Sliva ,D.(2005). Ganoderma lucidum suppresses angiogenesis through the inhibition of secretion of VEGF and TGF-1 from prostate cancer cells. Biochem Biophys Res Commun.;330:46-52.

Cheung ,P.C.K.(1996). The hypocholesterolemic effect of extracellular polysaccharidefrom the submerged fermentation of mushroom. Nutri. Res. ;16:1953-1957.

Bae, J.T.; Sinha, J.; Park, J.P.; Song, C.H and Yun, J.W. (2000). Optimization ofsubmerged culture conditions for exo-biopolymer production by Paecilomyces japonica. J. Microbiol. Biotechnol. 10: 482-487

Hwang, H.J.; Kim, S.W.; Xu, C.P.; Choi, J.W and Yun, J.W. (2003). Production and molecular characteristics of four groups of exopolysaccharides from submerged culture of Phellinus gilvus. J. Appl. Microbiol. 94: 708719

Nour, D.M.; Fallal, A.A.; Shahat, A.T. and Hereher, F.E.(2004). Exopolysaccharides production by Pleurotus pulmonarius: factors affecting formation and their structures. Pak. J. Biol. Sci. 7: 1078-1084.

Park, J.P.; Kim, S.W.; Hwang, H.J. and Yun, J.W. (2001). Optimization of submerged culture conditions for the mycelial growth and exobiopolymer production by Cordyceps militaris. Lett. Appl. Microbiol. 33: 76-81.

Wu, J.Z.; Peter, C.K.; Wong, K.H. and Huang, N.L. (2003). Studies on submerged fermentation of Pleurotus tuberregium (Fr.) Singer-Part 1: physical and chemical factors affecting the rate of mycelial growth and bioconversion efficiency. Food Chem. 81: 389-393

Rong, L. Xiao, L.J. and Guan, H.S. (2010). Optimization of mycelium biomass and exopolysaccharides production by Hirsutella $s p$. In submerged fermentation and evaluation of exopolysaccharides antibacterial activity. African Journal of Biotechnology Vol. 9 (2), pp. 195202. 
Leung, P.H.; Zhao,S.; Ho,K.P. and Wu,J.Y.(2009).Chemical properties and antioxidant activity of exopolysaccharide from mycelia culture of Cordyceps sinensis fungus CS-HK1. Food Chemistry, 114:1251-1256.

Gao, H. and Gu, W.Y. (2007). Optimization of polysaccharide and ergostrol production from Agaricus brasiliensis by fermentation process. Biochemical Engineering Journal, 33:202-210.

Xiao, J.H.; Chen, D.X.; Wan, W.H.; Hi, X.J.; Ying, Q. and Liang, Z.Q. ( 2006). Enhanced simultaneous production of mycelia and intracellular polysaccharide in submerged cultivation of Cordyceps jiangxiensis using desirability functions. Process biochemistry, 41: 1887-1893.

Yang,F.C. and Liau, C.B. (1998). The influence of environmental conditions on polysaccharide formation by Ganoderma lucidum in submerged cultures. Process Biochemistry, 33:547-553.

Bolton, W. and Blair,R. (1982). Poultry nutrition (Ministry of agriculture, fisheries and food reference book174) $\left(4^{\text {th }}\right.$ ed). London: Her Majesty's Stationery Office (pp.115121).

Pokhrel,C.P. and Ohga,S. (2007). Synthetic cultivation of Lyophyllum decastes on a combination of livestock compost and corn cob. Mushroom Science and Biotechnology,15.

Xu, C.P.; Kim, S.W.; Hwang, H.J.; Choi, J.W. and Yun, J.W. (2003). Optimization of submerged culture conditions for mycelial growth and exo- Biopolymer production by Paecilomyces tenuipes C240. Process Biochemistry, 38:1025-1030. 